\title{
Supportive Care Needs in Females With Breast Cancer Under Chemotherapy and Radiotherapy and its Predictors
}

\author{
Fatemeh Mirzaei $^{\circledR}$, Roghaiyeh Nourizadeh ${ }^{1}$, Shahla Hemmatzadeh $^{2}$, Reza Eghdam Zamiri ${ }^{3}$, \\ Azizeh Farshbaf-Khalili ${ }^{*}{ }^{*}$
}

\begin{abstract}
Objectives: The present study aimed to determine the unfulfilled needs of patients with breast cancer and the predictors of this disease in order to plan for appropriate interventions based on these needs.

Materials and Methods: This cross-sectional study was conducted on 190 females with breast cancer in Arak-Iran, who were selected by the convenience sampling method. Patients were evaluated by Socio-demographic and Disease Questionnaire, Perceived Illness Questionnaire, and Supportive Care Needs Survey Short Form. Data were analysed using SPSS, one-way ANOVA, chi-square, independent $t$ test, the Pearson correlation coefficient, and multivariate linear regression.

Results: Based on the results, the average number of supportive care needs was 100 (31.9). In addition, the greatest needs of patients were related to psychological, health-information, and physical sub-scales with a mean of 31.3 (12.5), 36.0 (12.5), 14.9 (5.8), respectively. Finally, multivariate linear regression analysis showed that family income and the perception of illness were among the predictors of supportive care needs.

Conclusion: In general, health attention providers are required to pay particular attention to the psychological, health, witting, and physical needs of females with mamma cancer and the related predictive factors of this disease.

Keywords: Supportive care, Care needs, Breast cancer
\end{abstract}

\section{Introduction}

Breast cancer is now regarded as the main health concern since it is the most prevalent type of cancer among females and the other leading reason of cancer death after lung cancer (1). In addition, it is responsible for one in five deaths in women aged 40-50 (2). Breast cancer accounted for around 1.8 million deaths worldwide in 2013 (3). Having a chronic disease, especially cancer, provokes and creates countless needs in cancer patients, their families, and caregivers (4). According to the report of the American Cancer Society, providing optimal care and treatment services to cancer patients requires examination and recognition of their needs (5). Therefore, assessing the needs is a strategy for identifying the unresolved concerns experienced by these patients and determining their needs in order to help them in various parts of the care chain. Further, the needs of every patient should be followed to achieve good and efficient quality care (6) and assessing the needs of cancer patients should cover all important aspects of their life such as physical, emotional, and spiritual dimensions, as well as social functions (7). Previous studies regarding the needs of females with breast cancer referred to different cancer patients' requirements like high levels of educational needs and information, the need for psychological support to reduce the concerns of the patients and their family members about the progression or recurrence of the disease in addition to the need for sexual counseling and assistance with daily homework (8-11). Addressing the unfulfilled needs of the cancer patients is essential since neglecting their needs during different stages of the treatment is associated with mental and psychological complications, physical loss, and a reduced quality of life in the individual, which can itself have a negative impact on the patients' health system due to the overuse of the healthcare services and increased costs (12). Regardless the great prevalence of breast cancer, few studies have investigated the supportive attention needs of women with breast cancer in Iran while not considering the stage of the disease or the kind of received therapy ( 9 , 13,14). Therefore, timely identification and estimation of their needs in different stages of therapy seem necessary for modifying the quality of living of those females with breast cancer. Accordingly, the present research sought to identify the supportive surveillance needs of women with mamma cancer who underwent chemotherapy and radiotherapy referring to oncology centers in Arak. 
Determining the unfulfilled needs of these females among various cultures and societies and during different stages of treatment can help plan for stage-based interventions based on the needs, and ultimately, modify the quality of life of the patients.

\section{Materials and Methods}

Study Plan and Subjects

The current cross-sectional study was performed during June-July 2017. The research population included all females with breast cancer who referred to public and private oncology centers in Arak (Iran) for chemotherapy or radiotherapy. The inclusion criteria, obtained from medical records, included suffering from breast cancer irrespective of its stage, being within the age range of 1860 years, undergoing chemotherapy or radiotherapy with no record of chronic or systemic disease, having no history of psychiatric disorder or other cancers at the same time, and not undergoing chemotherapy or radiotherapy due to the relapse of the disease. Furthermore, the exclusion criteria were patients with breast cancer diagnosed in less than a month and the occurrence of stressful events (e.g., divorce, the relatives' death, or job loss) over the past six months. The sample size was estimated at 176 subjects using the following formula by a confidence interval of $95 \%, \mathrm{~m}=38.16, \mathrm{SD}=27.15, \alpha=0.05$, and $\mathrm{d}=0.075$ (9). Considering a possible drop of $10 \%$ in the sample, 190 subjects entered the study.

$$
n=\frac{Z_{1-\frac{\alpha}{2}}^{2} \delta^{2}}{d^{2}}
$$

\section{Sampling}

The convenience sampling method was used to select the study subjects. After approving the study by the Ethics Committee of the Tabriz College of Medical Sciences (IR.TBZMED.REC.1395.965) in 2016, the researcher investigated the subjects in terms of the inclusion and exclusion criteria and then provided them with data about the research purpose and method, along with the confidentiality of the information. The subjects were then requested to participate in the study and written informed consent was obtained if the patients showed a willingness for participation in the study. The study subjects included 190 women with breast cancer who were receiving chemotherapy or radiotherapy. Moreover, they completed a socio-demographic and disease questionnaire, a perceived illness questionnaire, and short-form supportive care needs survey questionnaire (SCNS-SF34) in an appropriate setting. In the case of fatigue or unsuitable physical status of the subjects, the questionnaires were completed at other times depending on their willingness and convenience. In addition, data related to the type and stage of cancer and the type of the received drugs were collected from the patient file.
Instruments

The socio-demographic and disease questionnaire included information concerning individual and disease characteristics.

Additionally, SCNS-SF34 was designed by Boyes in 2003 and encompassed 34 items that addressed several dimensions such as the supportive needs in the somatic and daily life (5 items), psychological (10 items) and patient surveillance and support (5 items), health system and information (11 items), and sexual matters (3 items). The subjects responded to each section based on a 5-point Likert-type scale including no need-no use, the lack of need fulfillment, low need, average need, and high need ranging from 1 to 5 , respectively. A higher number reflected higher needs. Boyes et al reported a Cronbach alpha coefficient of above 0.8 and $r>0.55$ (15). In addition, Rahmani et al found a Cronbach alpha coefficient of above 0.9 and tool stability of 0.9 for the Persian version of the questionnaire (9).

Further, illness perception questionnaire, developed by Broadben et al, was used to measure the patients' perceptions of their disease. This questionnaire contained 9 questions that assessed the emotional and cognitive perceptions of the disease. A high score indicated a great deal of concern about the disease and the scores ranged from 0 to 80 . The Cronbach alpha coefficient and the stability of the original version of the instrument were 0.80 and 0.75 , respectively (16). Furthermore, Bagherian et al reported the Cronbach alpha coefficient of 0.80 and the test-retest reliability of $0.42-0.75$ for various domains within 6 weeks for the Persian version of the tool (17).

\section{Statistical Analysis}

Data were analyzed using SPSS software, version 21.0. Moreover, the Pearson test, independent $t$ test, and one-way ANOVA were initially utilized for analyzing the descriptive data and analytical statistics including frequency distribution and mean (standard deviation). Then, the independent variables with a $P$ value of less than 0.2 in the bivariate tests were entered the multivariate linear regression model using backward strategy in order to determine the predictors of supportive care needs after controlling the confounding variables. Eventually, linear regression assumptions including linear relationship, multivariate normality, no or little collinearity, no autocorrelation, and homoscedasticity were investigated before multivariate analysis.

\section{Results}

Table 1 demonstrates the demographic characteristics of breast cancer sufferers and their relationship with supportive care needs. As shown, the mean (SD) age and the average of the number of children is 46.9 (8.8) years and 2.8 (1.4), respectively. Most subjects (95.4\%) are insured and half of them have low-income families (50\%). Additionally, about $83.1 \%$ of the patients are housewives 
Table 1. Demographic Characteristics of Breast Cancer Sufferers and Their Relationship With Supportive Care Needs

\begin{tabular}{|c|c|c|c|}
\hline Demographic Characteristics & No. (\%) & $\begin{array}{c}\text { SCNs } \\
\text { Mean (SD) }\end{array}$ & $P$ Value \\
\hline Age/mean (SD) & $46.9(8.8)$ & $100.2(31.9)$ & $0.400^{\mathrm{a}}$ \\
\hline Number of children/mean (SD) & $2.8(1.4)$ & 100.2 (31.9) & $0.805^{\mathrm{a}}$ \\
\hline Husband's age/mean (SD) & $51.9(9.9)$ & $100.2(31.9)$ & $0.584^{\mathrm{a}}$ \\
\hline \multicolumn{4}{|l|}{ Insurance } \\
\hline Yes & $184(95.4)$ & $99.6(32.0)$ & \multirow[t]{2}{*}{$0.144^{b}$} \\
\hline No & $6(4.6)$ & $119.0(21.1)$ & \\
\hline \multicolumn{4}{|l|}{ Job } \\
\hline Housewife & $157(83.1)$ & $102.5(30.6)$ & \multirow{4}{*}{$0.256^{c}$} \\
\hline Working at home & $4(2.1)$ & $98.8(37.5)$ & \\
\hline Working outdoors & $17(9.0)$ & $89.9(36.6)$ & \\
\hline Retired & $11(5.8)$ & $88.5(38.3)$ & \\
\hline \multicolumn{4}{|l|}{ Education } \\
\hline Illiterate & 36 (18.9) & $102.0(30.4)$ & \multirow{4}{*}{$0.378^{d}$} \\
\hline Elementary/guidance & $76(40)$ & $101.9(30.3)$ & \\
\hline High school/Diploma & $48(25.3)$ & $102.2(34.9)$ & \\
\hline Academic & $30(15.8)$ & $90.8(32.2)$ & \\
\hline \multicolumn{4}{|l|}{ Income status } \\
\hline Relatively sufficient & $77(40.5)$ & $91.5(33.7)$ & \multirow{3}{*}{$0.006^{d}$} \\
\hline Sufficient & $18(9.5)$ & 101.4 (30.9) & \\
\hline Insufficient & $95(50.0)$ & $107.1(29.1)$ & \\
\hline \multicolumn{4}{|l|}{ Marital status } \\
\hline Married & $157(82.7)$ & 100.2 (31.9) & \multirow{4}{*}{$0.437^{c}$} \\
\hline Unmarried & $15(7.9)$ & $91.8(38.5)$ & \\
\hline Divorced & $5(2.6)$ & $95.8(31.1)$ & \\
\hline Widow & $13(6.8)$ & $111.3(22.9)$ & \\
\hline \multicolumn{4}{|l|}{ Husband's education } \\
\hline Illiterate & $23(14.4)$ & $103.3(34.4)$ & \multirow{4}{*}{$0.200^{d}$} \\
\hline Elementary/guidance & $65(40.7)$ & $100.7(29.6)$ & \\
\hline High school/diploma & $40(25.0)$ & $107.4(31.7)$ & \\
\hline Academic & $32(20.0)$ & $91.4(33.3)$ & \\
\hline \multicolumn{4}{|l|}{ Address } \\
\hline Town & 167 (87.9) & $101.4(32.2)$ & \multirow[t]{2}{*}{$0.150^{\mathrm{b}}$} \\
\hline Village & $23(12.1)$ & $91.3(28.5)$ & \\
\hline
\end{tabular}

SCNs: Supportive care needs; SD: Standard deviation.

The range of scores for support needs (34-170). In the demographic profile, all items except for the specified cases are expressed by the number (percent).

a Pearson Correlation; ${ }^{b}$ Fisher exact test; ${ }^{c}$ chi-square test; ${ }^{d}$ Linear by linear chi-square.

and $40 \%$ of them have primary and secondary education. The disease characteristics of breast cancer patients and their relationship with supportive care needs are represented in Table 2. Based on the data, most participants $(143,76.5 \%)$ undergo mastectomy and carcinoma in situ and grade 2 carcinoma account for the most common type of disease in women with $58.4 \%$ and $53.7 \%$, respectively. In addition, the mean (SD) score of supportive care needs
Table 2. The Disease Characteristics of the Breast Cancer Sufferers and Their Relationship With Supportive Care Needs

\begin{tabular}{|c|c|c|c|}
\hline $\begin{array}{l}\text { Characteristics } \\
\text { of Disease }\end{array}$ & No. (\%) & $\begin{array}{l}\text { SCNs } \\
\text { Mean (SD) }\end{array}$ & $P$ Value \\
\hline \multicolumn{4}{|l|}{ Type of breast disease } \\
\hline Carcinoma in situ & $111(58.4)$ & $96.6(30.9)$ & \multirow[t]{2}{*}{$0.065^{\mathrm{a}}$} \\
\hline Invasive & $79(41.6)$ & $105.3(32.7)$ & \\
\hline \multicolumn{4}{|l|}{ Stage of illness } \\
\hline Grade 1 & $14(7.4)$ & $89.5(25.2)$ & \multirow{4}{*}{$0.080^{\mathrm{b}}$} \\
\hline Grade 2 & $102(53.7)$ & $101.0(32.8)$ & \\
\hline Grade 3 & $64(33.6)$ & $97.8(30.2)$ & \\
\hline Grade 4 & $10(5.3)$ & $122.1(35.4)$ & \\
\hline \multicolumn{3}{|l|}{$\begin{array}{l}\text { How to know about the } \\
\text { disease }\end{array}$} & \multirow{5}{*}{$0.651^{a}$} \\
\hline Doctor & $142(74.7)$ & $99.7(31.8)$ & \\
\hline Nurse & $1(0.5)$ & $102.0(0.0)$ & \\
\hline Relatives & 18) 9.5) & $94.5(29.7)$ & \\
\hline Others & $29(15.3)$ & $106.2(43.3)$ & \\
\hline \multicolumn{4}{|l|}{ Type of treatment } \\
\hline Chemotherapy & $92(48.4)$ & $97.6(30.5)$ & \multirow[t]{2}{*}{$0.273^{\mathrm{a}}$} \\
\hline Radiotherapy & $98(51.6)$ & $102.7(33.1)$ & \\
\hline \multicolumn{3}{|l|}{ Surgical type } & \multirow{3}{*}{$0.680^{\mathrm{a}}$} \\
\hline Mastectomy & $143(76.5)$ & 100.7 (31.9) & \\
\hline Lumpectomy & $44(23.5)$ & $98.4(33.3)$ & \\
\hline $\begin{array}{l}\text { Past time of diagnosis } \\
\text { (month)/ mean (SD) }\end{array}$ & $6.9(2.8)$ & 100.2 (31.9) & $0.696^{c}$ \\
\hline $\begin{array}{l}\text { Past time of surgery } \\
\text { (month)/mean (SD) }\end{array}$ & $5.4(2.9)$ & $100.2(31.9)$ & $0.368^{c}$ \\
\hline Perception Illness/mean (SD) & $38.9(13.1)$ & $100.2(31.8)$ & $0.552^{c}$ \\
\hline
\end{tabular}

in the chemotherapy and radiotherapy groups and the overall score are 97.6 (30.5), 102.7 (33.1), and 100.2 (31.9), respectively (within the range of 34-170). As regards the domains of supportive surveillance needs, higher scores indicate the unfulfilled needs in that domain. Based on the data provided in Table 3, the highest supportive care needs are reported by patients in three domains including the psychological aspect with a mean (SD) of $31.3(12.5)$ out of the score range of 10-50, the health information system with a mean of 36 (12.5) out of the score range of 11-55, and the physical domain with a mean of 14.9 (5.8) out of the score range of 5-25. Comparing the outcomes of the independent $t$-test, there was no important difference among the patients of chemotherapy and radiotherapy groups in terms of the overall score of supportive surveillance needs $(P>0.05)$ while an important diversity was observed between the two groups regarding the domain of Information and health system $(P=0.030)$.

The results show a significant correlation between supportive surveillance needs and income status (Table 
Table 3. The Mean Score of Supportive Surveillance Needs and its Dimensions in Females With Breast Cancer Under Chemotherapy and Radiotherapy

\begin{tabular}{|c|c|c|c|c|c|c|c|}
\hline Domains & $\begin{array}{c}\text { Chemotherapy } \\
(n=92) \text { Mean (SD) }\end{array}$ & $\begin{array}{c}\text { Radiotherapy } \\
(n=98) \text { Mean (SD) }\end{array}$ & $\begin{array}{l}\text { Total Mean } \\
\text { (SD) }\end{array}$ & $\begin{array}{c}\text { Attainable } \\
\text { Score Range }\end{array}$ & $\begin{array}{l}\text { Number of } \\
\text { Questions }\end{array}$ & $\begin{array}{l}\text { Min-Max } \\
\text { Scoring }\end{array}$ & $P$ Value \\
\hline Physical & $15.0(5.7)$ & $14.7(5.8)$ & $14.9(5.8)$ & $5-25$ & 5 & $5-25$ & $0.770^{\mathrm{a}}$ \\
\hline Psychological & $31.7(12.2)$ & $31.4(12.8)$ & $31.3(12.5)$ & $10-50$ & 10 & $10-50$ & $0.898^{\mathrm{a}}$ \\
\hline Care and support of patients & $10.0(6-14)^{c}$ & $11.5(7-16)^{c}$ & $11.6(5.5)$ & $5-25$ & 5 & $5-25$ & $0.191^{b}$ \\
\hline Information and health system & $35.0(26-44.7)^{c}$ & $42.5(27.7-49)^{c}$ & $36.0(12.5)$ & $11-55$ & 11 & $11-55$ & $0.030^{\mathrm{b}}$ \\
\hline Sexual & $5.0(3-9)^{c}$ & $6.0(3-9)^{c}$ & $6.4(3.6)$ & 3-15 & 3 & $3-15$ & $0.519^{b}$ \\
\hline Total score & $97.6(30.4)$ & $102.7(33.1)$ & 100.2 (31.9) & $34-170$ & 34 & $34-163$ & $0.273^{\mathrm{a}}$ \\
\hline
\end{tabular}

andependent sample $t$ test; ${ }^{\mathrm{b}}$ Mann-Whitney test; 'Median (Q25-Q75) are reported. In addition, higher scores indicate more unmet needs in the intended domain.

1). As shown, unmet needs are noted with a higher mean of 107.1 (29.1) in individuals with poor economic status. Further, the mean (SD) score of the women's perception of their cancer is 38.9 (13.1) from the score range of $0-80$ and a significant relationship is found between illness perception and supportive care needs (Table 2). In other words, the unmet need is higher in those with a high perception of the disease. Based on the results of Table 4 , income status and illness perception are still the main predictors of supportive care needs even after entering the variables (with a $P<0.2$ ) into the multivariate linear regression model and examining the interaction between such variables. Income and perception of illness accounted for $33 \%$ of the variance in the supportive care needs of females with breast cancer.

\section{Discussion}

The present research mainly aimed to determine the supportive care needs of females with mamma cancer undergoing chemotherapy and radiotherapy. The outcomes of the current research showed that most of the sufferers who participated in this study reported psychological, information and safety system, and physical domains as their greatest needs. Consistent with the results of the present study, Rahmani et al conducted a study investigating the unfulfilled needs in breast cancer sufferers in Tabriz and found that most unmet needs of the patients were related to the health information system and somatic-everyday life domains (9). Similarly, another study in Switzerland reported psychological and emotional domains as the most unmet needs in these women (18). In another study on the most unmet needs of cancer patients

Table 4. Predictors of Supporting Surveillance Needs in Females With Breast Cancer

\begin{tabular}{lcc}
\hline Variable & $\boldsymbol{\beta}$ (Cl 95\%) & $\boldsymbol{P}$ \\
\hline Income & Reference & - \\
Insufficient & $-12.1(-19.75$ to -4.52$)$ & 0.002 \\
Relatively sufficient & $-8.71(-22.83$ to 5.41$)$ & 0.225 \\
Sufficient & $1.31(1.03$ to 1.60$)$ & $<0.001$ \\
Perception of illness & $\mathbf{0 . 3 3}$ \\
Adjusted $\mathrm{R}^{2}$ & \multicolumn{2}{c}{} \\
\hline
\end{tabular}

who suffered from the stomach, intestine, lung, and other cancers, Ramezanzade et al (19) referred to financial concerns and occupational issues as the most frequent unsatisfied needs of cancer patients. Such a difference may be due to the fact that $51.1 \%$ of the participants in this study were men who shouldered the economic burden of the family. Similarly, in the review study on cancer sufferers (i.e., breast, lung, prostate, colorectal, and cervix) in Nigeria, Okediji et al found the highest needs in the information-health system, as well as psychological and physical-everyday life domains (20). Furthermore, Olson et al, as well as Park et al referred to the patients' need for information about their disease as the most unmet need in both urban and rural patients $(21,12)$. Moreover, Moradi et al highlighted the information needs as the most significant need of females with breast cancer in Tabriz, Iran (22) while Uchida et al reported information-health and psychological needs as the most important needs of women with breast cancer (11). The results of several studies in Southwest Asia demonstrated that health and information needs are more frequently observed among the other supportive care providers (23-25). Additionally, based on the results of a comparative study, health need was found to be the most frequent need in females with breast cancer in Hong Kong while physical, everyday life, and psychological needs were reported as the most requested needs among German women (26). Conversely, the results of the studies performed in Western countries indicated the health and data system domains as the second or third need $(27,28)$. Thus, supportive care needs seem to be culture-dependent (26) and the needs may be perceived differently by individuals in different cultures.

Patients with breast cancer face multiple psychological problems as well (18). The results of previous studies show that individuals with a degree of unsatisfied psychological needs have a superior level of unsatisfied needs in the bodily and workaday activity dimension $(19,24)$. Individuals with cancer have psychological needs in every phase of the illness and patients' willingness for supportive surveillance describes these needs as one of the most important factors in coping with their disease (29). As a result, psychological disorders can 
have a profound negative effect on performance status, the modality of living, the length of stay, and even the remedy outcomes for cancer sufferers (30). Therefore, combining the psychological components of cancer care and the healthcare strategy are considered effective. Thus, healthcare providers should actively and continuously provide patients with information about the condition of their disease and treatment that is consistent with the need for emotional and psychological care throughout the treatment process. In addition, increased physical and daily life needs during active treatment may be due to the side effects associated with treatment or delay in the treatment.

The results of the present study revealed a significant relationship between illness perception and supportive care needs while no study was found to confirm or reject these findings. Some Iranian researchers reported that many cancer patients were unaware of their disease prognosis and considered cancer as a treatable disease $(31,32)$. Accordingly, focusing on the variations in illness perception should be highlighted as an effective factor in the supportive surveillance needs of women with cancer in subsequent studies.

In the current research, there was a reverse relationship between income status and supportive care needs, which is in line with the outcomes of several previous studies $(19,20,23)$, therefore, strengthening the insurance coverage for cancer patients in the country is of great importance (33). Moreover, providing timely and appropriate healthcare services as well as training and information on the disease and complications of treatment and rehabilitation programs should be regarded as the most important priorities in supporting the patients.

Due to the unwillingness to exclude poor-educated and illiterate women, the researcher collected the required data by completing the questionnaires through interview. Questioning the patients undergoing chemotherapy and radiotherapy was one of the main limitations of this study. These patients were not in a good psychological state and did not easily communicate with the interviewers. To resolve this problem, sampling was conducted in a quiet environment, as much as possible, after explaining the goals, assuring the patients of confidentiality, and creating an empathic mutual relationship. In addition, the present research was performed in one geographical area and thus the results cannot be generalized to other contexts or cultures. Therefore, conducting future studies concerning comparing the supportive care needs between different areas and cultures is a necessity. Finally, the cause-effect relationship cannot be expressed between the variables since the present study is not of interventional type.

\section{Conclusions}

Generally speaking, breast cancer sufferers encounter many treatment-related, as well as disease management problems and complications. Therefore, considering the diverse needs and concerns of patients with mamma cancer, accurate recognition of their needs and concerns is highly essential. Further, understanding the needs of the sufferers help healthcare providers to offer counseling interventions and supportive services for patients and their families. Based on the results of this study, Iranian peace vigilance providers need to focus on the psychological, health information, and physical needs of females with mamma cancer. Furthermore determining the unmet needs of such women in various cultures and societies and through different stages of treatment can help plan for stage-based interventions based on women's needs, and ultimately, modify their quality of life.

\section{Conflict of Interests}

Authors declare that they have no conflict of interests.

\section{Financial Support}

This research was financially supported by the Tabriz College of Medical Sciences.

\section{Acknowledgments}

This paper is based on a research project approved by the Vice-chancellor of the Research of Tabriz College of Medical Sciences. We would like to express our gratitude to all those who helped us with this research including the Vice-chancellor of Research of Tabriz College of Medical Sciences, the Tabriz Faculty of Nursing and Midwifery, the Vice-chancellor for Treatment of Arak College of Medical Sciences, and other colleagues at Ayatollah Khansari Hospital and Imam Reza Clinic of Arak. Moreover, we would like to affirm our gratuity to all the women and their families for their participation in the study. Special thanks go to Ms. Rosa Mobayen from University College London for her contribution in native English editing of this article.

\section{References}

1. Mahamoud A. Breast cancer screening in racialized women: implications for health equity. Wellesley Institute; 2014.

2. Yen JY, Ko CH, Yen CF, et al. Quality of life, depression, and stress in breast cancer women outpatients receiving active therapy in Taiwan. Psychiatry Clin Neurosci. 2006;60(2):147-153. doi:10.1111/j.1440-1819.2006.01479.x

3. Fitzmaurice C, Dicker D, Pain A, et al. The global burden of cancer 2013. JAMA Oncol. 2015;1(4):505-527. doi:10.1001/ jamaoncol.2015.0735

4. Rahnama M, Fallahi Khoshknab M, Seyed Bagher Madah S, Ahmadi F. Cancer patients' perception of spiritual care. J Med Ethics Hist Med. 2012;5(3):64-80. [Persian].

5. Harrison JD, Young JM, Price MA, Butow PN, Solomon MJ. What are the unmet supportive care needs of people with cancer? A systematic review. Support Care Cancer. 2009;17(8):1117-1128. doi:10.1007/s00520-009-0615-5

6. Sanson-Fisher R, Girgis A, Boyes A, Bonevski B, Burton L, Cook P. The unmet supportive care needs of patients with cancer. Supportive Care Review Group. Cancer. 
2000;88(1):226-237.

7. Schmid-Buchi S, Halfens RJ, Dassen T, van den Borne B. Psychosocial problems and needs of posttreatment patients with breast cancer and their relatives. Eur J Oncol Nurs. 2011;15(3):260-266. doi:10.1016/j.ejon.2009.11.001

8. Taleghani F, Bahrami M, Loripoor M, Yousefi A. Empowerment needs of women with breast cancer: a qualitative study. Iran Red Crescent Med J. 2014;16(11):e16379. doi:10.5812/ircmj.16379

9. Abdollahzadeh F, Moradi N, Pakpour V, et al. Un-met supportive care needs of Iranian breast cancer patients. Asian Pac J Cancer Prev. 2014;15(9):3933-3938.

10. So WK, Chow KM, Chan HY, et al. Quality of life and most prevalent unmet needs of Chinese breast cancer survivors at one year after cancer treatment. Eur J Oncol Nurs. 2014;18(3):323-328. doi:10.1016/j.ejon.2014.03.002

11. Uchida M, Akechi T, Okuyama T, et al. Patients' supportive care needs and psychological distress in advanced breast cancer patients in Japan. Jpn J Clin Oncol. 2011;41(4):530536. doi:10.1093/jjco/hyq230

12. Park BW, Hwang SY. Unmet needs and their relationship with quality of life among women with recurrent breast cancer. J Breast Cancer. 2012;15(4):454-461. doi:10.4048/ jbc.2012.15.4.454

13. Safaee A, Zeighami B, Tabatabaee H, Moghimi Dehkordi B. Quality of life and related factors in breast cancer patients under chemotherapy. Iran J Epidemiol. 2008;3(3):61-66. [Persian].

14. Leilabady L, Nassiri Ziba F, Nazari Geirani M, Hosseini F. Patients with breast cancer and their needs. Iran Journal of Nursing. 2005;17(40):28-38. [Persian].

15. Boyes A, Girgis A, Lecathelinais C. Brief assessment of adult cancer patients' perceived needs: development and validation of the 34-item Supportive Care Needs Survey (SCNS-SF34). J Eval Clin Pract. 2009;15(4):602-606. doi:10.1111/j.1365-2753.2008.01057.x

16. Broadbent E, Petrie KJ, Main J, Weinman J. The brief illness perception questionnaire. J Psychosom Res. 2006;60(6):631637. doi:10.1016/j.jpsychores.2005.10.020

17. Bagherian Sararoodi R, Afshar H, Forouzandeh N, et al. The relationship between illness perception and symptom severity in patients with irritable bowel syndrome. Journal of Isfahan Medical School. 2011;29(137):2-5. [Persian].

18. Edib Z, Kumarasamy V, Binti Abdullah N, Rizal AM, AlDubai SA. Most prevalent unmet supportive care needs and quality of life of breast cancer patients in a tertiary hospital in Malaysia. Health Qual Life Outcomes. 2016;14:26. doi:10.1186/s12955-016-0428-4

19. Ramezanzade Tabriz E, Parsa Yekta Z, Shahsavari H, Yekaninejad MS. Unmet needs and its relationship with the management of depression in patients suffering from cancer. Iran J Nurs Res. 2015;9(4):12-21. [Persian].

20. Okediji PT, Salako O, Fatiregun OO. Pattern and predictors of unmet supportive care needs in cancer patients. Cureus. 2017;9(5):e1234. doi:10.7759/cureus.1234
21. Olson RA, Howard F, Turnbull K, et al. Prospective evaluation of unmet needs of rural and aboriginal cancer survivors in Northern British Columbia. Curr Oncol. 2014;21(2):e179-185. doi:10.3747/co.21.1729

22. Moradi N, Abdollahzadeh F, Rahmani A, Zamanzadeh V, Asvadi I, Ghaleban K. Effects of husbands' education on meeting supportive care needs of breast cancer patients: A randomized clinical trial. Sci J Hamdan Univ Med Sci. 2013;21(3):40-50. [Persian].

23. Schmid-Buchi S, Halfens RJ, Muller M, Dassen T, van den Borne B. Factors associated with supportive care needs of patients under treatment for breast cancer. Eur J Oncol Nurs. 2013;17(1):22-29. doi:10.1016/j.ejon.2012.02.003

24. Li WW, Lam WW, Au AH, et al. Interpreting differences in patterns of supportive care needs between patients with breast cancer and patients with colorectal cancer. Psychooncology. 2013;22(4):792-798. doi:10.1002/ pon. 3068

25. Okuyama T, Akechi T, Yamashita H, et al. Oncologists' recognition of supportive care needs and symptoms of their patients in a breast cancer outpatient consultation. Jpn J Clin Oncol. 2011;41(11):1251-1258. doi:10.1093/jjco/ hyr 146

26. Lam WW, Au AH, Wong JH, et al. Unmet supportive care needs: a cross-cultural comparison between Hong Kong Chinese and German Caucasian women with breast cancer. Breast Cancer Res Treat. 2011;130(2):531-541. doi:10.1007/ s10549-011-1592-1

27. Sanders SL, Bantum EO, Owen JE, Thornton AA, Stanton AL. Supportive care needs in patients with lung cancer. Psychooncology. 2010;19(5):480-489. doi:10.1002/ pon. 1577

28. 28. Malekian A, Alizadeh A, Ahmadzadeh GH. Anxiety and depression in cancer patients. Journal of Research in Behavioural Sciences. 2007;5(2):115-119.

29. Rajabizadeh G, Mansoori SM, Shakibi MR, Ramazani MR. Determination of factors related to depression in cancer patients of the oncology ward in Kerman. Journal of Kerman University of Medical Sciences. 2005;12(2):142147. [Persian].

30. Liao MN, Chen SC, Chen SC, et al. Changes and predictors of unmet supportive care needs in Taiwanese women with newly diagnosed breast cancer. Oncol Nurs Forum. 2012;39(5):E380-389. doi:10.1188/12.onf.e380-e389

31. Larizadeh $\mathrm{MH}$, Malekpour Afshar R. Knowledge of patients with cancer towards their disease status. Research in Medicine. 2007;31(1):85-90.

32. Schmid-Buchi S, Halfens RJ, Muller M, Dassen T, B. VdB. Factors associated with supportive care needs of patients under treatment for breast cancer. Eur J Oncol Nurs. 2013;17(1):22-9.

33. Rahmani A. Hope inspiring proces among Iranian cancer patients [dissertation]. Tabriz: Tabriz Nursing and Midwifery faculty; Tabriz University of Medical Sciences, Iran; 2011. [Persian].

(c) 2019 The Author (s); This is an open-access article distributed under the terms of the Creative Commons Attribution License (http://creativecommons.org/licenses/by/4.0), which permits unrestricted use, distribution, and reproduction in any medium, provided the original work is properly cited. 